\title{
Ultrasound and Cystic Echinococcosis
}

\section{(C) (1) $\odot$}

Authors

Enrico Brunetti ${ }^{1}$, Francesca Tamarozzi ${ }^{2}$, Calum Macpherson ${ }^{3}$, Carlo Filice ${ }^{1}$, Markus Schindler-Piontek ${ }^{4}$, Adnan Kabaalioglu ${ }^{5}$, Yi Dong ${ }^{6}$, Nathan Atkinson ${ }^{7}$, Joachim Richter ${ }^{8}$, Dagmar Schreiber-Dietrich ${ }^{9}$, Christoph F Dietrich ${ }^{10}$

Affiliations

1 San Matteo Hospital Foundation, University of Pavia, Unit of Infectious and Tropical Diseases, Pavia, Italy

2 Center for Tropical Diseases, Sacro Cuore-Don Calabria Hospital, Negrar, Verona, Italy

3 St.George's University, Ultrasound, Grenada, India

4 Caritas Krankenhaus Bad Mergentheim, Academic Teaching Hospital of the University of Würzburg, Medical Clinic 2, Bad Mergentheim, Germany

5 Akdeniz University, Radiology, Antalya, Turkey

6 Zhongshan Hospital, Ultrasound, Shanghai, China

7 John Radcliffe Hospital, Oxford University Hospitals NHS Trust, Department of Gastroenterology, Oxford, New Zealand

8 Institute of Tropical Medicine and International Health, Charité Universitätsmedizin, Berlin, Germany

9 Caritas-Krankenhaus Bad Mergentheim, Department of Pediatrics, Bad Mergentheim, Germany

10 Caritas-Krankenhaus, Medizinische Klinik 2, Bad Mergentheim, Germany

Key words

puncture- aspiration-injection-re-aspiration (PAIR),

liver cysts, albendazole, guidelines

received 25.10.2017

revised 25.05.2018

accepted $\quad 18.06 .2018$

Bibliography

DOI https://doi.org/10.1055/a-0650-3807

Ultrasound Int Open 2018; 4: E70-E78

(c) Georg Thieme Verlag KG Stuttgart · New York

ISSN 2199-7152
Correspondence

Dr. Christoph F.Dietrich

Caritas-Krankenhaus

Medizinische Klinik 2

Uhlandstr. 7

97980, Bad Mergentheim

Germany

Tel.: +49/7931/58 2201, Fax: +49/7931/58 2290

Christoph.Dietrich@ckbm.de

\section{ABSTRACT}

The introduction of imaging techniques in clinical practice 40 years ago changed the clinical management of many diseases, including cystic echinococcosis (CE). For the first time cysts were clearly seen before surgery. Among the available imaging techniques, ultrasound (US) has unique properties that can be used to study and manage cystic echinococcosis. It is harmless, can image almost all organs and systems, can be repeated as often as required, is portable, requires no patient preparation, is relatively inexpensive and guides diagnosis, treatment and follow-up without radiation exposure and harm to the patient. US is the only imaging technique which can be used in field settings to assess CE prevalence because it can be run even on solar power or a small generator in remote field locations. Thanks to US classifications, the concept of stage-specific treatments was introduced and because US is repeatable, the scientific community has gained a clearer understanding of the natural history of the disease. This paper reviews the scope of US in CE, describes its strengths and weaknesses compared to other imaging techniques and its relationship with serodiagnosis and discusses sonographic features that may be helpful in differential diagnosis.

\section{Introduction}

Noninvasive visualization of cystic lesions in the body has revolutionized the management of cystic echinococcosis (CE). Ultrasound (US) provides the clinician with important clinical information including the location, number, size and stage of cysts, with a higher sensitivity and specificity than serology. Portable US scanners have for the first time facilitated population screening. This screen- ing allowed assessment of the true prevalence of disease in remote rural communities [1-3]. Currently US is the best method to assess the prevalence of CE due to the peculiar biological features of this parasitic disease in humans, to its portability and its acceptance by communities throughout the world. Furthermore, US is superior to CT or MRI for staging cysts [4]. 


\section{Cystic echinococcosis}

Cystic echinococcosis, also known as hydatid disease or hydatidosis, is an infection caused by the larval stage (metacestode) of the cestode Echinococcus granulosus. In humans it may result in a wide spectrum of clinical manifestations ranging from asymptomatic infection to severe, even fatal disease.

E. granulosus has a broad geographic range and occurs on all continents including circumpolar, temperate, subtropical, and tropical zones. The highest prevalence of the parasite is found in parts of Eurasia, Africa, Australia, and South America.

Even within endemic zones, there is variation from high prevalence to sporadic infection, but only a few countries can be regarded as being free of $E$. granulosus.

Echinococcal cysts are found in the liver in approximately $70 \%$ of cases, and the lungs in approximately $25 \%$ of cases. The spleen, kidney, heart, muscle, bone and central nervous system are involved less frequently [5].

In each anatomic site, cysts are surrounded by periparasitic host tissue (pericyst), which encompasses the larval endocyst. The endocyst has an outer, acellular laminated layer and an inner, or germinative, layer that gives rise to brood capsules and protoscolices.

The cyst is filled with clear fluid, numerous brood capsules and protoscolices. Cysts may also harbor daughter vesicles of variable size. Data is scarce regarding the growth and natural history of echinococcal cysts. The growth rate of the cysts is variable, with cyst diameter thought to increase on average $1 \mathrm{~cm}$ per year. Observational studies and unpublished experience gathered in referral centers suggest the natural history of CE. Changes to cyst structure occur in stages, which tend towards inactivity in a process that is favorable to the host. Early unilocular cysts (stage CE1) progress through stage CE3a to solidification of the cyst (CE4). Reactivation from stage CE3a can produce CE2 cysts, while reactivation from stage CE4 produces CE3b cysts. This has important consequences for screening and treatment, as CE2 and CE3b are generally non-responsive to non-surgical approaches.

Serious complications include mechanical complications due to mass effect either as compression of bile ducts with secondary cholestasis (common), or compression of vessels causing portal hypertension or Budd-Chiari syndrome (very rare). Liver infections can spread to the peritoneum with secondary echinococcosis and lung infections to pleural cavity in case of cyst rupture and spillage [6]. Cysts can rupture into the biliary system (common). Although rarely observed, anaphylactic shock can result from traumatic or other rupture of the cyst $[7,8]$.

\section{How are echinococcal cysts diagnosed?}

The diagnosis of $\mathrm{CE}$ is mainly made using imaging methods. For those cysts without pathognomonic signs, the adjunctive use of serology may be helpful. E. granulosus eggs are shed in feces passed by the definitive hosts, canids, but not by intermediate hosts. Therefore, direct parasitological diagnosis in humans is only possible through demonstration of viable protoscolices in the cyst, which can be obtained at surgery or by percutaneous aspiration. The latter cannot be performed routinely for technical and safety reasons, although the risk of anaphylaxis has been greatly exaggerated [9].
Although US is the modality of choice for determining cyst stage and number and the extent of disease [10], CT and MRI are valuable in certain circumstances previously described in detail $[5,11-$ 13] and expanded upon below. US is the cornerstone of diagnosis, staging and follow-up of CE [6].

\section{Ultrasound}

US is the imaging modality of choice due to its availability, lack of radiation and high resolution for the diagnosis, staging, differential diagnosis and follow-up of most abdominal cystic lesions $[12,14,15]$. Moreover, it has an established role in the interventional treatment of $C E[4,6]$. For field surveys, portable ultrasound is important as a screening tool [16-18].

\section{Computed tomography (CT)}

$\mathrm{CT}$ is indicated when US is unsatisfactory, particularly in obese patients or when visualization is difficult due to gas or bone $[6,19]$. CT should also be considered to evaluate postoperative changes, suspicion of abdominal spillage, for better visualization of calcifications, in the event of air within the cyst and suspicion of biliary communication (MRI is the method of choice before endoscopic or surgical treatment). Historically $\mathrm{CT}$ has been the leading cross-sectional imaging method in the diagnosis and treatment of $\mathrm{CE}$, but today MRI has been accepted as superior in many circumstances. CT provides invaluable clues for the assessment of complications and is indispensable in the diagnostic phase, for evaluating lung and bone. It is also important when there is diagnostic uncertainty on ultrasound, in planning surgical intervention, and diagnosing recurrent disease $[19,20]$. Unenhanced CT is the modality of choice to assess calcifications. Calcification is not limited to inactive late stages but may be present in all stages, including, although only to a limited extent, early stages [13]. Contrast-enhanced CT is crucial in the differential diagnosis of focal liver lesions [21]. CT can also be used to guide PAIR in specific cases where US may be insufficient or provides inadequate visualization for intervention [12, 22]. PAIR stands for puncture, aspiration, injection of a scolecidal solution and reaspiration [23-25].

\section{Magnetic Resonance Imaging (MRI)}

MR cholangiography is preferred in complicated cases of communication or rupture into the biliary system. Additionally, MRI may be indicated when US is insufficient and CT is contraindicated. MRI has a high sensitivity for the detection of CE, particularly to assess the cyst number, size, location and relations to neighboring structures. MRI is indicated in patients in whom sonographic visualization is impaired because of bowel gas, obesity or previous surgical interventions, in disseminated disease, extra-abdominal location and complications. MRI is also useful for pre-surgical evaluation and follow-up [5, 26]. MRI with a T2-weighted sequence is better than $\mathrm{CT}$ for characterizing the internal structures of echinococcal cysts, reproducing better the ultrasound-defined features of CE [4]. MRI better visualizes the liquid areas inside the matrix $[11,26]$ and should be preferred for pre-treatment assessment whenever possible. MR cholangiography is as sensitive as endoscopic retrograde cholangiography (ERC) to evaluate cysto-biliary communications although it cannot be used for interventional procedures $[26,27]$. 
Conventional X-ray

Portable X-ray equipment has been used in field studies to look at the prevalence of deep-seated lung cysts that are inaccessible to US [28], but they are rarely diagnosed in such surveys [28, 29].

\section{Serology}

Serologic tests are useful for confirming a presumptive imaging diagnosis. However, the limitations of sero-diagnosis in CE must be borne in mind to correctly interpret results. Moreover, it must be emphasized that serology should not be used alone for the diagnosis of CE in the absence of a compatible lesion identified by imaging, as the positive predictive value of sero-diagnosis is low [30]. Many variables influence the performance of sero-diagnostic tests. These include test-related factors (antigens used, assay technique), patient-related factors (immune status) and cyst-related factors (location, stage, size, number, previous therapy and complications) $[31,32]$. Antigens used for the serological diagnosis of CE are not standardized, accounting for the extreme variability in reported diagnostic performance and the difficulty in comparing results from different groups. Generally speaking, tests based on hydatid cyst fluid (HCF) show a better sensitivity (sens. 80-99\% and spec. 60-97\%), while tests based on purified or recombinant proteins show a better specificity (sens. 38-93\% and spec. 80-100\%) $[33,34]$. False-negative test results may occur in cases of hepatic CE with young CE1 cysts (30-58\%), inactive CE4-CE5 cysts (50$87 \%$ ), and in cases of extra-hepatic CE; including up to $50 \%$ of patients with lung cysts and patients with cysts in other locations. Patients with active and transitional cysts (CE2, CE3a, CE3b) show lower sero-negativity rates (5-20\%), and patients with multiple cysts are generally sero-positive [31, 32, 35, 36]. Although cysts are classified as active (CE1, CE2, CE3b), transitional (CE3a) and inactive (CE4, CE5), the loss of integrity of the cyst structure (either spontaneous or as a consequence of therapy) rather than the biological viability (i. e. cyst activity) per se correlates with the presence of positive serology [31]. Serotiters are usually observed to increase in the months after medical or percutaneous treatments associated with disruption of cyst integrity, and slowly decrease over months or years after successful treatment [32, 37-39]. Serology may remain positive for years even after successful surgical treatment, limiting the use of serology to assess response to treatment, and leading the clinician to erroneously assume active infection and therefore to overtreat. Nonetheless, observing serotiters decrease over time (months to years) after treatment, or in the presence of inactive cysts, may provide an indication of cure [3739]. Similarly, antibody titers generally increase upon relapse, although not universally $[40,41]$. Assays detecting specific antibody classes or a number of recombinant proteins have been suggested to improve follow-up evaluation, [39, 40, 42, 43]. However, no such test is commercially available. False-positive results may occur in persons with other helminthic infections, especially in alveolar echinococcosis (AE) due to infection with E. multilocularis (50-100\%) The different band pattern in HCF-based western blot may discriminate between $E$. granulosus and E. multilocularis in about $75 \%$ of cases [44]. However, more specific tests for $E$. multilocularis infection should be applied in case of high suspicion. Less frequently, false positives can be seen with other non-infectious diseases, such as cancer and chronic immune disorders $[39,45,46]$.

\section{Ultrasound classification}

There are various classifications of the sonographic appearance of CE, the first and most widely used being proposed by Gharbi in 1981 [47]. In 2003, the World Health Organization Informal Working Group on Echinococcosis (WHO-IWGE) proposed a standardized US classification based on the active-transitional-inactive status of the cyst as suggested by its sonographic appearance [14].

The standardized classification scheme is intended to promote uniform standards of diagnosis and treatment and may be applied to the clinical treatment of patients as well as to field diagnostic surveys. In this classification, six cyst stages have been assigned to three clinical groups:

1. The 'active' group includes developing cysts, which may be unilocular (CE1) or multi-vesicular with daughter vesicles (CE2) and which are usually found to be viable.

2. The 'transitional' group (CE3) includes both cysts with detachment of endocyst (CE3a) and predominantly solid cysts with daughter vesicles (CE3b).

3. The 'inactive' group (CE4 and CE5) exhibits involution and solidification of cyst content with increasing degrees of calcification and are nearly always found to be non-viable.

The WHO classification provides a rational basis for choosing an appropriate CE treatment scheme and follow-up, i. e. surgery, percutaneous treatment such as PAIR, benzimidazole chemotherapy or simply 'watch \& wait'. The WHO classification recognizes two basic types of morphology for CE3: the 'water-lily sign' for floating membranes, which is now known as subclass CE3a, and predominantly solid cysts with daughter vesicles, or subclass CE3b. This subdivision has been proposed based on their different morphology and response to PAIR and albendazole, which is generally good for CE3a and poor for CE3b. A study using magnetic resonance spectroscopy has shown that these two subgroups have different metabolic profiles. Specifically, the metabolic profile of CE3b cysts is similar to that of viable (e.g.CE1 and CE2) stages, while cysts staged as CE3a can be either active or inactive. Importantly these results parallel studies examining biological viability evaluated microscopically after cyst removal [11].

\section{WHO Classification as an improved version of Gharbi classification}

WHO-IGWE classification allows a grouping of cysts into active, transitional, and inactive, which is relevant for treatment planning and follow-up [6]. In this classification, CE1 and CE2 are active cysts, the $\mathrm{CE} 3$ group represents the transitional cysts with CE3b being biologically active [11], while CE4 and CE5 groups are inactive, late stage cysts [14]. Importantly, Gharbi classification did not distinguish CE3b from CE4 cysts, which hampers a stage-specific approach to treatment. Another useful addition to Gharbi classification [47] is the " $\mathrm{CL}$ " category, indicating undifferentiated "cystic lesions' that require further investigations before a definitive diagnosis can be made. Compared to Gharbi, the WHO-IWGE reverses the order of CE2 and CE3, subgrouping the CE3 lesions $[6,14,48]$. CE1 and CE3a are considered to be early stages [14].

$\mathrm{CL}$, as a potentially parasitic cyst, needs to be differentiated from non-parasitic cysts. This may also happen with CE1 cysts and CE3a [18]. 
The WHO CE classification does not describe nor include terminology for the sequence of cyst involution seen spontaneously or induced by treatment [18].

\section{The WHO panels in detail}

The CE1 stage - Fig. 1 refers to a simple round or oval unilocular cyst with anechoic content and a visible double cystic wall. In early stages when the cysts are smaller than $4-5 \mathrm{~cm}$ and especially in children, the thick walls may not be seen. Therefore, differential diagnosis with simple liver or kidney cysts may sometimes be difficult.

The CE2 cyst is completely filled with daughter vesicles. What appears as "septa" are not true septa but the cyst walls of the daughter vesicles adjacent to one another $>$ Fig. 2 .

CE3 cysts includes two stages, CE3a and CE3b, which differ in terms of morphology, viability and clinical characteristics. CE3a is characterized by the "water-lily" sign, represented by floating membranes, i. e. the endocyst detached from the cyst outer wall (pericyst) $>$ Fig. 3. CE3b is a predominantly solid lesion with daughter vesicles $>$ Fig. 4 and $>$ Fig. 5. CE3a may go on to become "solid" (inactive) or may give rise to daughter vesicles, in which case it becomes a CE2 cyst.

US typically reveals coarse variable (hyper, hypo) echogenic echotexture without daughter vesicles. The "ball of wool" sign, corresponding to the detached endocyst as a hypoechoic folded structure embedded in a hyperechoic matrix, is the key US sign > Fig. 6. However, often a definitive diagnosis of CE in this stage cannot be made by US findings alone. If CE4 stage is reached spontaneously, these cysts tend to remain inactive over time and, if asymptomatic, need only US monitoring > Fig. 7 [41].

CE5 cysts are partially (with an egg-shell calcified wall) or completely calcified with shadowing. These cysts are not viable in the vast majority of cases. Definitive diagnosis cannot be made by ultrasound findings alone $>$ Fig. 8.

The " $\mathrm{CL}$ " category indicates an undifferentiated 'cystic lesion' that requires further investigations before a definitive decision is made about their parasitic nature. As such, strictly speaking, $C L$ is not a "stage" but rather a temporary label assigned to a cyst whose parasitic nature is still undefined. This is very helpful in ultrasound surveys in endemic areas when, for instance, the results of serological tests are still pending $\triangleright$ Fig. $\mathbf{9}$.

\section{US classification and serology: matches, mismatches and what to do about it}

The diagnosis of CE is mostly indirect and is based on imaging and serology. However, serology has several drawbacks as previously discussed: lack of standardization, cross-reactivity and antigen-dependent performance $[33,49,50]$, which depends also on cyst location and viability.

After ultrasound detection of a cyst with features compatible with CE but with no clear pathognomonic signs, a combination of diagnostic tests is recommended for confirmation (indirect hemagglutination (IHA), indirect immunofluorescence (IFAT), enzyme-linked immunosorbent assay (ELISA), and immunoblotting (IB)) [33]. In clinical practice, two tests are usually performed: ELISA (the more commonly used) and IHA. When results are inconclusive,

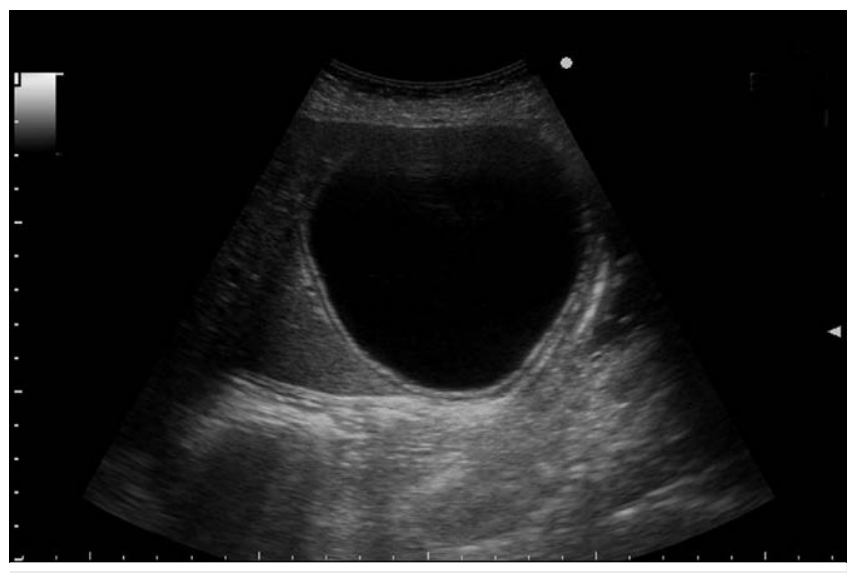

-Fig. 1 Appearance of a stage CE1 cyst. Ultrasound scan clearly demonstrates the double wall sign, pointing to the parasitic nature of the lesion.

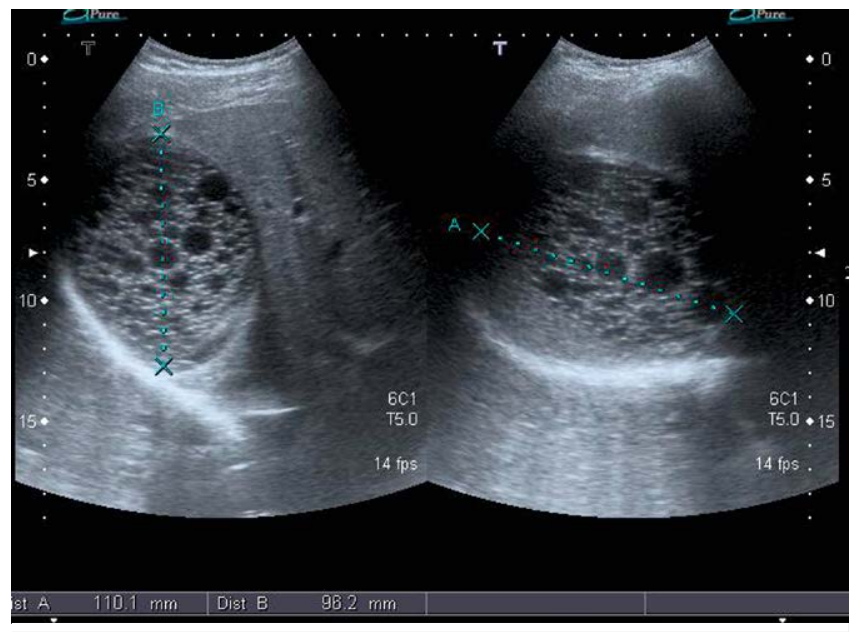

- Fig. 2 22-year-old man with CE2 cyst in the liver. The cyst is completely filled with daughter vesicles.

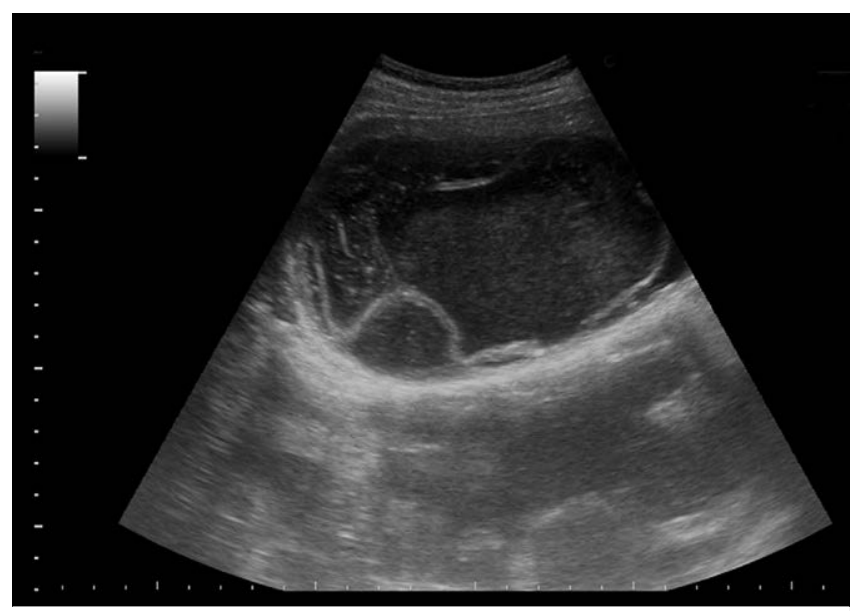

- Fig. 3 Appearance of a stage CE3a cyst. Ultrasound demonstrates the detached, folded endocyst. 


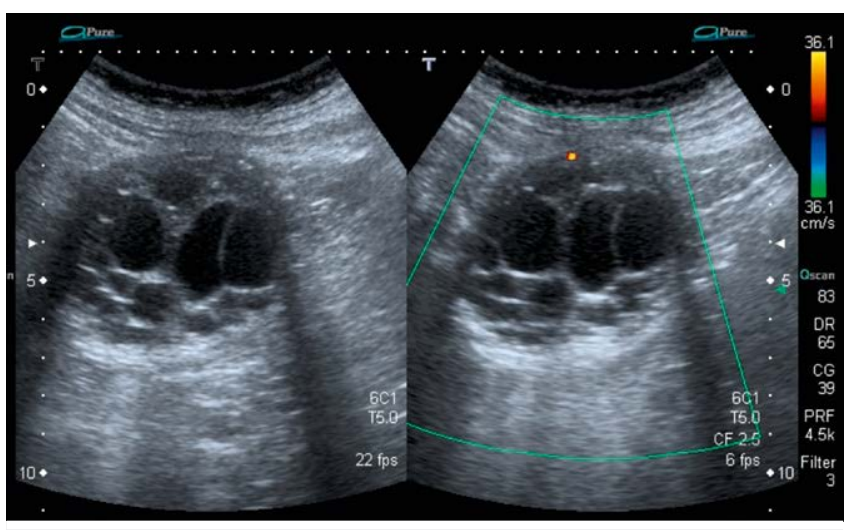

- Fig. 4 A CE3b hydatid cyst in the right liver lobe of a 77-year-old man who has been followed for more than two years.

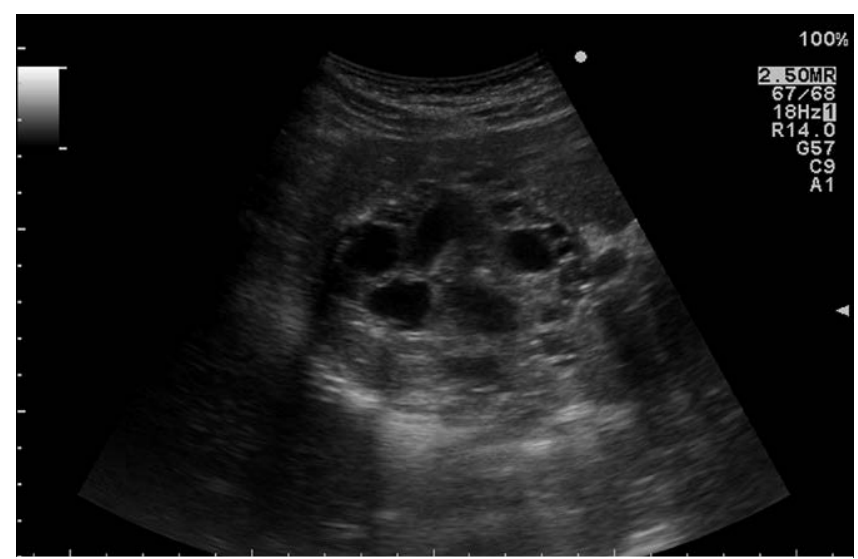

-Fig. 5 Appearance of a stage CE3b cyst. Ultrasound scan reveals multiple daughter vesicles within the cyst.

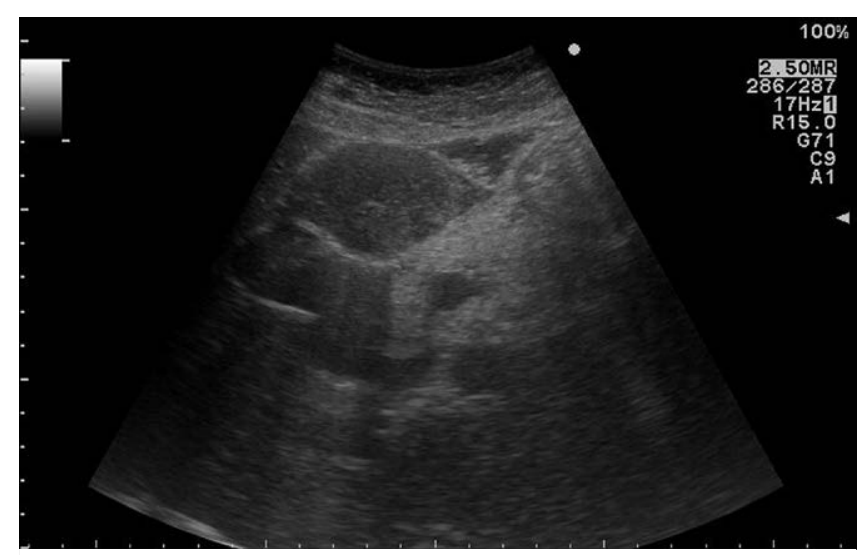

- Fig. 6 Appearance of a stage CE4 cyst. Ultrasound scan shows the cyst content is uniformly echogenic.
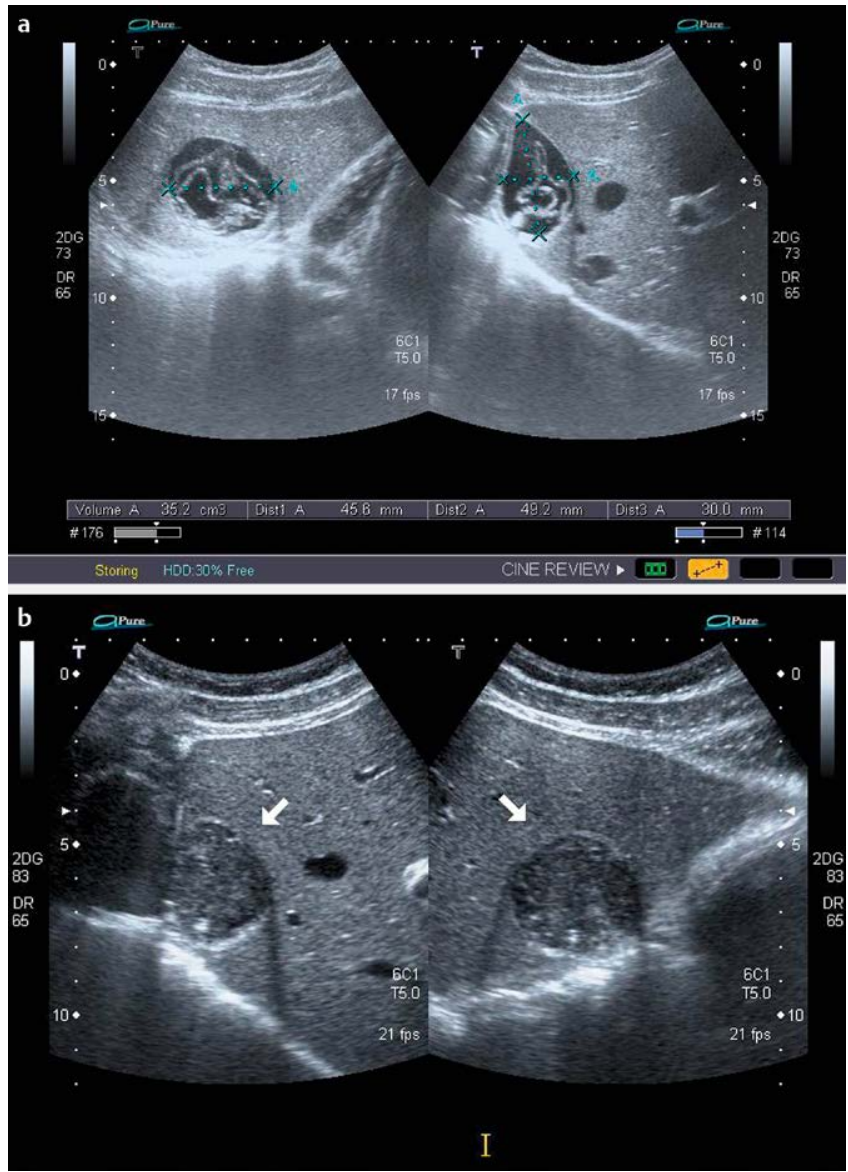

- Fig. 7 A 21-year-old male was referred for PAIR. The echinococcal cyst in the right liver lobe cyst in stage CE3a was smaller than $5 \mathrm{~cm}$ (approximately $35 \mathrm{cc}$ ) therefore treated with albendazole (a). After almost 6 years, the lesion was slightly smaller $(30 \mathrm{cc})$ and had solidified (b).

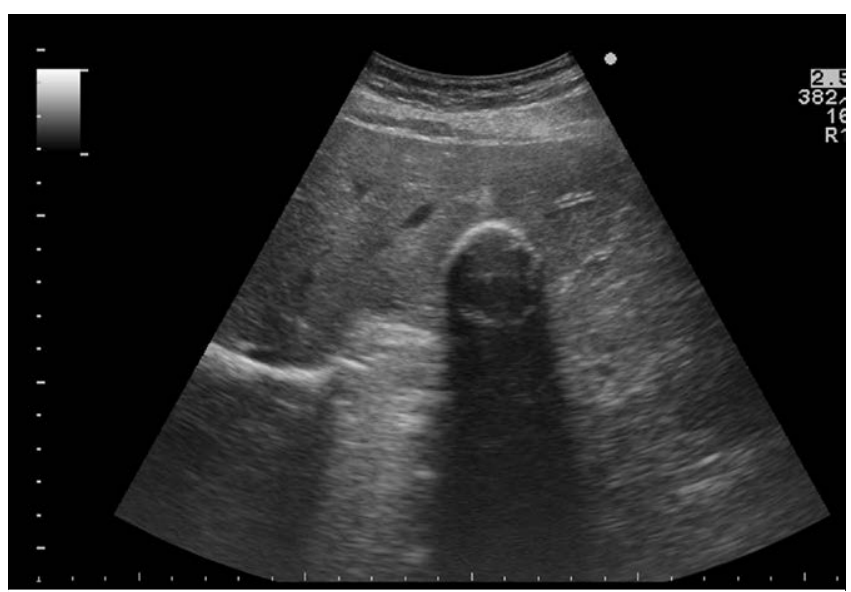

- Fig. 8 Appearance of a stage CE5 cyst. Ultrasound image of a CE5 cyst with the calcified rim clearly seen, together with a posterior acoustic shadowing. 


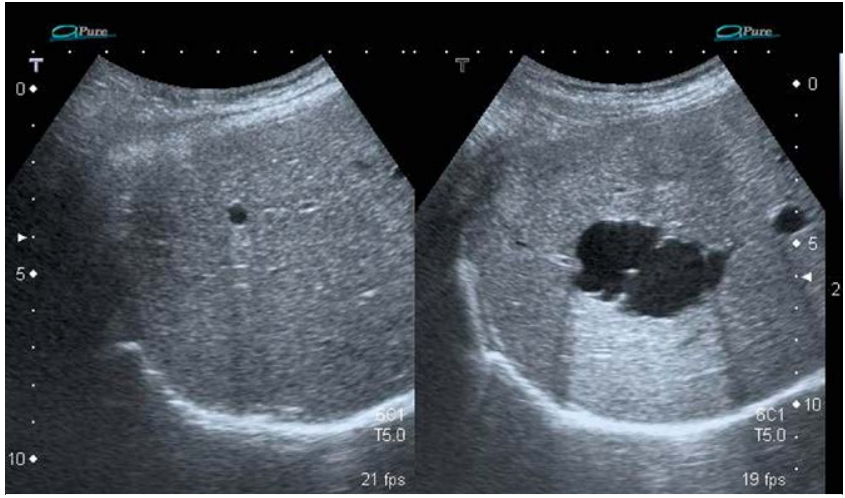

- Fig. 9 Ultrasound shows 2 liver cysts ( $\mathrm{CL}$ to the right) in a 43-yearold woman. $\mathrm{CL}$ is not a "stage" but rather a temporary label assigned to a cyst whose parasitic nature is still undefined.

IB can be performed as an additional test [5, 51-53]. Because of the high rates of false-negative results, especially in very early (CE1) and final stage cysts (CE4 and CE5), the role of serology is only confirmatory [18]. Serological testing in the context of liver involvement is more sensitive than for extrahepatic infections. The sensitivity of serological tests appears to be inversely related to the degree of sequestration of echinococcal antigens [10].

Furthermore, current serology tests are not designed to clearly distinguish between active and inactive CE. In practice, problems arise mostly with early CE1 and late CE4/5 stages. The inverse problem can be faced in patients with inactive cysts, who should have negative serology but often are positive $[41,50]$. Positive serology in these cases may be misleading when the patient has previously been treated.

\section{Differential diagnosis of parasitic liver lesions}

Echinococcal cysts have to be differentiated from other conditions, such as non-parasitic cysts, single or multiple hemangiomas, pyogenic or amoebic liver abscesses, hematoma, and neoplasia with hemorrhage and necrosis (e. g., large adenoma, hepatocellular carcinoma, metastases, lymphoma), biloma and post-surgical sequelae and textiloma $[5,54,55]$. Most frequently, simple cysts are encountered but atypical cysts sometimes pose a diagnostic challenge. These include biliary cysts, polycystic liver disease, mucinous cystic neoplasms (cystic (biliary) adenoma, cystadenoma), cystic metastases and other very rare diseases, e. g., ciliated hepatic foregut cysts. Additionally other infectious agents must be considered: fungal, bacterial and amoebic abscesses [10]. In most uncertain cases, diagnosis should be achieved using aspiration. Only under particular circumstances, small and very large (> $50 \mathrm{~mm}$ ), asymptomatic and uncomplicated simple cysts, may be monitored. This can be done by serial ultrasound at six-month intervals for the first two years following diagnosis. Significant growth, the development of progressive symptoms, or any suspicion of neoplastic change requires a definite diagnosis and surgical intervention. Other parasitic liver manifestations occasionally need to be considered in the differential diagnosis [56-58].

Determining whether a cystic lesion is echinococcal depends on the presence of a double wall and is obvious when membrane de- tachment is present. Simple or minimally complex cysts, as well as biliary cystadenocarcinomas or abscesses, lack these features.

\section{Treatment}

Ultrasound has a crucial role in the treatment of CE as a widely used means of guidance for percutaneous treatments. US is also crucial in the evaluation of treatment response (see below), and for assessing for inactivity in CE4 and CE5 asymptomatic liver cysts when managed expectantly, i. e. the so-called "watch and wait" approach [41], due to the lack of ionizing radiation and repeatability.

PAIR is indicated for medium-sized CE1 and CE3a liver cysts $[5,48]$. Recent EFSUMB guidelines on ultrasound-guided procedures $[59,60]$ list several abdominal ultrasound-guided treatment options $[61,62]$.

CE2 and CE3b cysts are not responsive to PAIR $[48,63]$. Although daughter vesicles can be punctured individually, these stages show growth of new daughter vesicles in the weeks following a procedure $[5,48,64]$. Successful drainage of the entire cyst content via large bore catheters has been reported in centers with specific expertise, but studies with larger cohorts of patients are needed to compare these methods with surgery [65].

Before a PAIR procedure, the patient should have careful pre-procedure assessment. Albendazole should be started at least $4 \mathrm{~h}$ beforehand, as prophylaxis against secondary echinococcosis in case of inadvertent spillage of cystic fluid into the peritoneum, and continued for 1 month $[6,66]$.

The puncture can usually be made by a 20 -gauge fine needle but use of thinner or larger gauge needles has been reported [67]. Some authors use catheter drainage when cysts are bigger than $5-6 \mathrm{~cm}[68,69]$. Early studies on large-bore catheter evacuation of large cysts reported prolonged hospital stay and increased biliary complications [70]. However, catheter drainage is effective in selected cases, and when the required expertise is available [65]. If possible, a route through the hepatic parenchyma should be used to prevent peritoneal spillage of cyst contents. Usually all cystic content can be aspirated, before a scolicidal agent such as $96 \%$ ethanol or hypertonic (20\%) saline is injected into the cavity $[6,59,60]$. The amount of scolicidal agent should not exceed $1 / 3$ or $1 / 2$ of the initial cyst volume. For cysts larger than $600 \mathrm{cc}$, a maximum amount of $200 \mathrm{cc}$ is advised [6]. After 5-10 min, the fluid is re-aspirated [6].

The patient should have IV access during the procedure and vital parameters should be monitored by an anesthesiologist or by a certified anesthesia nurse. Medications for the urgent treatment of anaphylaxis should be readily available [6].

The cystic fluid is usually clear in early (CE1) cysts but the color may be dark yellow and the material viscous in later stages or infected cysts. The aspirated fluid should be examined under a microscope to assess for the presence of viable protoscolices [6].

Evaluation and management of cystic communication with the biliary tree is debated. Commercially available dipsticks can immediately determine the presence of bilirubin in the aspirate. Some experts prefer cystography - that is injecting contrast material into the cyst cavity - to establish whether the cyst has a biliary connection. Most authors suggest that if the aspirate is not clear-colorless but contains bile, then scolecidal agents should not be given [24]. 
Others argue that hypertonic saline may be given with caution, and to date no biliary damage related to PAIR has been reported [5].

In mid-sized CE1 and CE3a cysts, PAIR has an overall response rate $>80 \%$, while multi-vesiculated CE2 and CE3b cysts have a success rate of less than $40 \%$ [65]. However, randomized, placebo-controlled clinical trials on the use of PAIR are lacking. A Cochrane review on PAIR with or without albendazole for the treatment of uncomplicated hepatic CE could evaluate only two randomized clinical trials comparing PAIR with either albendazole treatment alone or surgery and no other randomized trial has been published since. Both trials were small (30 and 50 patients, respectively), but graded as "adequate", and demonstrated a significantly better efficacy and lower morbidity than that of the treatments with which they were compared. The authors conclude that "PAIR with or without benzimidazole coverage may be comparable or superior to surgery or medical treatment with benzimidazoles alone for uncomplicated hepatic hydatid cysts", although "data are not sufficient to draw definitive conclusions" [71].

After PAIR ultrasound, follow-up can be scheduled at one week, one month, three months, six months and then annually thereafter. CT may be necessary during follow-up and in cases with multiple cysts [71, 72]. US plays a crucial role in following the involution process resulting from treatment and in monitoring relapse (growth of new daughter vesicles) both after treatment and in the "watch and wait" approach [41].

\section{Conclusion}

Ultrasound allows diagnosis, differential diagnosis, treatment guidance and follow-up of CE. US has the additional role of a tool for mass screenings, which are currently the best way to assess the prevalence of CE in a population. Echinococcal cysts are predominantly observed in the liver where US is the best and easiest imaging modality. For lesions in the lungs, brain or other rare locations, CT and MRI are used. Although we have learned much from what US reveals and now have consensus on cyst types and stage-specific approach for hepatic cysts, the best treatment and follow-up algorithms remain a matter of debate.

\section{Acknowledgements}

This work was funded in part by the EU FP7 Program (HERACLES) under grant agreement $\mathrm{n}^{\circ} 602051$ (to E. Brunetti).

\section{Conflict of Interest}

The authors declare no conflict of interest.

\section{References}

[1] MacPherson CN, Romig T, Zeyhle E, Rees PH, Were JB. Portable ultrasound scanner versus serology in screening for hydatid cysts in a nomadic population. Lancet 1987; 2: 259-261

[2] Macpherson CN, Milner R. Performance characteristics and quality control of community based ultrasound surveys for cystic and alveolar echinococcosis. Acta Trop 2003; 85: 203-209
[3] Dietrich CF, Goudie A, Chiorean L, Cui XW, Gilja OH, Dong Y, Abramowicz JS et al. Point of Care Ultrasound: A WFUMB Position Paper. Ultrasound Med Biol 2017; 43: 49-58

[4] Stojkovic M, Rosenberger K, Kauczor HU, Junghanss T, Hosch W. Diagnosing and staging of cystic echinococcosis: How do CT and MRI perform in comparison to ultrasound? PLoS Negl Trop Dis 2012; 6: e1880

[5] Rinaldi F, Brunetti E, Neumayr A, Maestri M, Goblirsch S, Tamarozzi F. Cystic echinococcosis of the liver: A primer for hepatologists. World J Hepatol 2014; 6: 293-305

[6] Brunetti E, Kern P, Vuitton DA. Writing Panel for the WHO IWGE. Expert consensus for the diagnosis and treatment of cystic and alveolar echinococcosis in humans. Acta Trop 2010; 114: 1-16

[7] Murali MR, Uyeda JW, Tingpej B. Case records of the Massachusetts General Hospital. Case 2-2015. A 25-year-old man with abdominal pain, syncope, and hypotension. N Engl J Med 2015; 372: 265-273

[8] Popovic-Dragonjic L, Jovanovic M, Vrbic M, Kostic V, Miladinovic-Tasic N, Kocic B, Rankovic A et al. Anaphylactic shock due to unruptured hepatic hydatid cyst complicated by multiple intrahospital infections. Turkiye Parazitol Derg 2014; 38: 261-263

[9] Neumayr A, Troia G, de Bernardis C, Tamarozzi F, Goblirsch S, Piccoli L, Hatz $C$ et al Justified concern or exaggerated fear: The risk of anaphylaxis in percutaneous treatment of cystic echinococcosis-a systematic literature review. PLoS Negl Trop Dis 2011; 5: e1154

[10] Moro P, Schantz PM. Echinococcosis: a review. Int ] Infect Dis 2009; 13 : 125-133

[11] Hosch W, Junghanss T, Stojkovic M, Brunetti E, Heye T, Kauffmann GW, Hull WE. Metabolic viability assessment of cystic echinococcosis using high-field 1 H MRS of cyst contents. NMR Biomed 2008; 21: 734-754

[12] Hosch W, Junghanss T, Werner ], Dux M. [Imaging methods in the diagnosis and therapy of cystic echinococcosis]. Rofo 2004; 176: 679-687

[13] Hosch W, Stojkovic M, Janisch T, Kauffmann GW, Junghanss T. The role of calcification for staging cystic echinococcosis (CE). Eur Radiol 2007; 17: 2538-2545

[14] Group WHOIW. International classification of ultrasound images in cystic echinococcosis for application in clinical and field epidemiological settings. Acta Trop 2003; 85: 253-261

[15] Pawlowski ZS, Eckert J, Vuitton DA et al. WHO/OIE Manual on Echinococcosis in Humans and Animals: a Public Health Problem of Global Concern. In: Eckert J, Gemmell M, Meslin FX, Pawlowski ZS.eds. Office International des Epizooties (OIE). Paris: 2001: 20-72

[16] Moro PL, Garcia HH, Gonzales AE, Bonilla J], Verastegui M, Gilman RH. Screening for cystic echinococcosis in an endemic region of Peru using portable ultrasonography and the enzyme-linked immunoelectrotransfer blot (EITB) assay. Parasitol Res 2005; 96: 242-246

[17] Macpherson CN, Bartholomot B, Frider B. Application of ultrasound in diagnosis, treatment, epidemiology, public health and control of Echinococcus granulosus and E. multilocularis. Parasitology 2003; 127 (Suppl): S21-S35

[18] Brunetti E, Garcia HH, Junghanss T. on behalf of the members of the International Ce Workshop in Lima P. Cystic Echinococcosis: Chronic, Complex, and Still Neglected. PLoS Negl Trop Dis 2011; 5: e1146

[19] Richter ], Hatz C, Haussinger D. Ultrasound in tropical and parasitic diseases. Lancet 2003; 362: 900-902

[20] Derbel F, Ben Mabrouk M, Ben Hadj Hamida M, Mazhoud J. al. e. Hydatid Cysts of the Liver - Diagnosis, Complications and Treatment. In: Derbel F.(ed.) Abdominal Surgery: InTech. 2012

[21] Chiorean L, Caraiani C, Radzina M, Jedrzejczyk M, Schreiber-Dietrich D, Dietrich CF. Vascular phases in imaging and their role in focal liver lesions assessment. Clin Hemorheol Microcirc 2015; 62: 299-326

[22] Hosch W, Kauffmann GW, Junghanss T. [Cystic liver lesions with unspecified upper abdominal pain]. Radiologe 2005; 45: 924-928 
[23] Khuroo MS, Wani NA, Javid G, Khan BA, Yattoo GN, Shah AH, Jeelani SG. Percutaneous drainage compared with surgery for hepatic hydatid cysts. N Engl J Med 1997; 337: 881-887

[24] Dietrich CF, Nuernberg D. Interventional Ultrasound: A Practical Guide and Atlas. Stuttgart, New York, Delhi, Rio: Thieme publisher; 2014

[25] Filice C, Pirola F, Brunetti E, Dughetti S, Strosselli M, Foglieni CS. A new therapeutic approach for hydatid liver cysts. Aspiration and alcohol injection under sonographic guidance. Gastroenterology 1990; 98: 1366-1368

[26] Hosch W, Stojkovic M, Janisch T, Heye T, Werner ], Friess H, Kauffmann GW et al. MR imaging for diagnosing cysto-biliary fistulas in cystic echinococcosis. Eur.J.Radiol. 2008; 66: 262-267

[27] Ignee A, Baum U, Schuessler G, Dietrich CF. Contrast-enhanced ultrasound-guided percutaneous cholangiography and cholangiodrainage (CEUS-PTCD). Endoscopy 2009; 41: 725-726

[28] Gavidia CM, Gonzalez AE, Zhang W, McManus DP, Lopera L, Ninaquispe $\mathrm{B}$, Garcia $\mathrm{HH}$ et al. Diagnosis of cystic echinococcosis, central Peruvian Highlands. Emerg Infect Dis 2008; 14: 260-266

[29] Macpherson CN, Kachani M, Lyagoubi M, Berrada M, Shepherd M, Fields PF, El Hasnaoui M. Cystic echinococcosis in the Berber of the Mid Atlas mountains, Morocco: new insights into the natural history of the disease in humans. Ann Trop Med Parasitol 2004; 98: 481-490

[30] Torgerson PR, Deplazes P. Echinococcosis: Diagnosis and diagnostic interpretation in population studies. Trends Parasitol 2009; 25: 164-170

[31] Lissandrin R, Tamarozzi F, Piccoli L, Tinelli C, De Silvestri A, Mariconti $M$, Meroni $V$ et al. Factors Influencing the Serological Response in Hepatic Echinococcus granulosus Infection. Am J Trop Med Hyg 2015

[32] Hernandez-Gonzalez A, Santivanez S, Garcia HH, Rodriguez S, Munoz S, Ramos G, Orduna A et al. Improved serodiagnosis of cystic echinococcosis using the new recombinant 2B2t antigen. PLoS Negl Trop Dis 2012; 6: e1714

[33] Carmena D, Benito A, Eraso E. Antigens for the immunodiagnosis of Echinococcus granulosus infection: An update. Acta Trop 2006; 98: $74-86$

[34] Manzano-Roman R, Sanchez-Ovejero C, Hernandez-Gonzalez A, Casulli A, Siles-Lucas M. Serological Diagnosis and Follow-Up of Human Cystic Echinococcosis: A New Hope for the Future? Biomed Res Int 2015; 2015: 428205

[35] Barbieri M, Fernandez V, Gonzalez G, Luaces VM, Nieto A. Diagnostic evaluation of a synthetic peptide derived from a novel antigen $B$ subunit as related to other available peptides and native antigens used for serology of cystic hydatidosis. Parasite Immunol 1998; 20: 51-61

[36] Ortona E, Rigano R, Margutti P, Notargiacomo S, loppolo S, Vaccari S, Barca $S$ et al. Native and recombinant antigens in the immunodiagnosis of human cystic echinococcosis. Parasite Immunol 2000; 22: 553-559

[37] Li T, Ito A, Pengcuo R, Sako Y, Chen X, Qiu D, Xiao N et al. Post-treatment follow-up study of abdominal cystic echinococcosis in tibetan communities of northwest Sichuan Province, China. PLoS Negl Trop Dis 2011; 5: e1364

[38] Ben Nouir N, Nunez S, Gianinazzi C, Gorcii M, Muller N, Nouri A, Babba H et al. Assessment of Echinococcus granulosus somatic protoscolex antigens for serological follow-up of young patients surgically treated for cystic echinococcosis. J Clin Microbiol 2008; 46: 1631-1640

[39] Hernandez-Gonzalez A, Muro A, Barrera I, Ramos G, Orduna A, Siles-Lucas M. Usefulness of four different Echinococcus granulosus recombinant antigens for serodiagnosis of unilocular hydatid disease (UHD) and postsurgical follow-up of patients treated for UHD. Clin Vaccine Immunol 2008; 15: 147-153

[40] Lawn SD, Bligh J, Craig PS, Chiodini PL. Human cystic echinococcosis: Evaluation of post-treatment serologic follow-up by $\mathrm{lgG}$ subclass antibody detection. Am J Trop Med Hyg 2004; 70: 329-335
[41] Piccoli L, Tamarozzi F, Cattaneo F, Mariconti M, Filice C, Bruno A, Brunetti E. Long-term sonographic and serological follow-up of inactive echinococcal cysts of the liver: Hints for a "watch-and-wait" approach. PLoS Negl Trop Dis 2014; 8: e3057

[42] Ben Nouir N, Gianinazzi C, Gorcii M, Muller N, Nouri A, Babba H, Gottstein B. Isolation and molecular characterization of recombinant Echinococcus granulosus P29 protein (recP29) and its assessment for the post-surgical serological follow-up of human cystic echinococcosis in young patients. Trans R Soc Trop Med Hyg 2009; 103: 355-364

[43] Rigano R, loppolo S, Ortona E, Margutti P, Profumo E, Ali MD, Di Vico B et al. Long-term serological evaluation of patients with cystic echinococcosis treated with benzimidazole carbamates. Clin Exp Immunol 2002; 129: 485-492

[44] Liance M, Janin V, Bresson-Hadni S, Vuitton DA, Houin R, Piarroux R. Immunodiagnosis of Echinococcus infections: Confirmatory testing and species differentiation by a new commercial Western Blot. J Clin Microbiol 2000; 38: 3718-3721

[45] de la Rue ML, Yamano K, Almeida CE, lesbich MP, Fernandes CD, Goto A, Kouguchi $\mathrm{H}$ et al. Serological reactivity of patients with Echinococcus infections (E. granulosus, E. vogeli, and E. multilocularis) against three antigen B subunits. Parasitol Res 2010; 106: 741-745

[46] Li T, Ito A, Chen X, Sako Y, Qiu J, Xiao N, Qiu D et al. Specific IgG responses to recombinant antigen $B$ and em 18 in cystic and alveolar echinococcosis in china. Clin Vaccine Immunol 2010; 17: 470-475

[47] Gharbi HA, Hassine W, Brauner MW, Dupuch K. Ultrasound examination of the hydatic liver. Radiology 1981; 139: 459-463

[48] Junghanss T, da Silva AM, Horton J, Chiodini PL, Brunetti E. Clinical management of cystic echinococcosis: State of the art, problems, and perspectives. Am J Trop Med Hyg 2008; 79: 301-311

[49] Zhang W, McManus DP. Recent advances in the immunology and diagnosis of echinococcosis. FEMS Immunol Med Microbiol 2006; 47: 24-41

[50] Mariconti M, Bazzocchi C, Tamarozzi F, Meroni V, Genco F, Maserati R, Brunetti E. Immunoblotting with human native antigen shows stage-related sensitivity in the serodiagnosis of hepatic cystic echinococcosis. Am J Trop Med Hyg 2014; 90: 75-79

[51] Zhang W, Li ], McManus DP. Concepts in immunology and diagnosis of hydatid disease. Clin Microbiol.Rev. 2003; 16: 18-36

[52] Lorenzo C, Ferreira HB, Monteiro KM, Rosenzvit M, Kamenetzky L, Garcia HH, Vasquez Y et al. Comparative analysis of the diagnostic performance of six major Echinococcus granulosus antigens assessed in a double-blind, randomized multicenter study. J Clin Microbiol 2005; 43: $2764-2770$

[53] Ortona E, Rigano R, Buttari B, Delunardo F, loppolo S, Margutti P, Profumo $E$ et al. An update on immunodiagnosis of cystic echinococcosis. Acta Trop 2003; 85: 165-171

[54] Polat P, Kantarci M, Alper F, Suma S, Koruyucu MB, Okur A. Hydatid disease from head to toe. Radiographics 2003; 23: 475-494

[55] Cattaneo F, Graffeo M, Brunetti E. Extrahepatic textiloma long misdiagnosed as calcified echinococcal cyst. Case Rep Gastrointest Med 2013; 2013: 261685

[56] Dietrich CF, Kabaalioglu A, Brunetti E, Richter J. Fasciolosis. Z Gastroenterol 2015; 53: 285-290

[57] Richter ], Botelho MC, Holtfreter MC, Akpata R, El Scheich T, Neumayr A, Brunetti E et al. Ultrasound assessment of schistosomiasis. Z Gastroenterol 2016; 54: 653-660

[58] Richter ], Azoulay D, Dong Y, Holtfreter MC, Akpata R, Calderaro ], El-Scheich T et al. Ultrasonography of gallbladder abnormalities due to schistosomiasis. Parasitol Res 2016; 115: 2917-2924

[59] Dietrich CF, Lorentzen T, Appelbaum L, Buscarini E, Cantisani V, Correas JM, Cui XW et al. EFSUMB Guidelines on Interventional Ultrasound (INVUS), Part III - Abdominal Treatment Procedures (Long Version). Ultraschall Med 2016; 37: E1-E32 
[60] Dietrich CF, Lorentzen T, Appelbaum L, Buscarini E, Cantisani V, Correas JM, Cui XW et al. EFSUMB Guidelines on Interventional Ultrasound (INVUS), Part III - Abdominal Treatment Procedures (Short Version). Ultraschall Med 2016; 37: 27-45

[61] Sidhu PS, Brabrand K, Cantisani V, Correas JM, Cui XW, D'Onofrio M, Essig $M$ et al. EFSUMB Guidelines on Interventional Ultrasound (INVUS), Part II. Diagnostic Ultrasound-Guided Interventional Procedures (Long Version). Ultraschall Med 2015; 36: E15-E35

[62] Sidhu PS, Brabrand K, Cantisani V, Correas JM, Cui XW, D'Onofrio M, Essig M et al. EFSUMB Guidelines on Interventional Ultrasound (INVUS), Part II. Diagnostic Ultrasound-Guided Interventional Procedures (Short Version). Ultraschall Med 2015; 36: 566-580

[63] Giorgio A, de Stefano G, Esposito V, Liorre G, Di Sarno A, Giorgio V, Sangiovanni $V$ et al Long-term results of percutaneous treatment of hydatid liver cysts: A single center 17 years experience. Infection 2008; 36: $256-261$

[64] Golemanov B, Grigorov N, Mitova R, Genov J, Vuchev D, Tamarozzi F, Brunetti E. Efficacy and safety of PAIR for cystic echinococcosis: Experience on a large series of patients from Bulgaria. Am J Trop Med Hyg 2011; 84: 48-51

[65] Tamarozzi F, Vuitton L, Brunetti E, Vuitton DA, Koch S. Non-surgical and non-chemical attempts to treat echinococcosis: Do they work? Parasite 2014; 21: 75
[66] Brunetti E, Junghanss T. Update on cystic hydatid disease. Curr Opin Infect Dis 2009; 22: 497-502

[67] Giorgio A, Tarantino L, de Stefano G, Francica G, Mariniello N, Farella $\mathrm{N}$, Perrotta A et al Hydatid liver cyst: an 11-year experience of treatment with percutaneous aspiration and ethanol injection. J Ultrasound Med 2001; 20: 729-738

[68] Ustunsoz B, Akhan O, Kamiloglu MA, Somuncu I, Ugurel MS, Cetiner S. Percutaneous treatment of hydatid cysts of the liver: long-term results. AJR Am J Roentgenol 1999; 172: 91-96

[69] Men S, Yucesoy C, Edguer TR, Hekimoglu B. Percutaneous treatment of giant abdominal hydatid cysts: Long-term results. Surg Endosc 2006; 20: 1600-1606

[70] Schipper HG, Lameris JS, van Delden OM, Rauws EA, Kager PA. Percutaneous evacuation (PEVAC) of multivesicular echinococcal cysts with or without cystobiliary fistulas which contain non-drainable material: first results of a modified PAIR method. Gut 2002; 50: 718-723

[71] Nasseri-Moghaddam S, Abrishami A, Taefi A, Malekzadeh R. Percutaneous needle aspiration, injection, and re-aspiration with or without benzimidazole coverage for uncomplicated hepatic hydatid cysts. Cochrane Database Syst Rev 2011; CD003623

[72] Kabaalioglu A, Ceken K, Alimoglu E, Apaydin A. Percutaneous imaging-guided treatment of hydatid liver cysts: Do long-term results make it a first choice? Eur J Radiol 2006; 59: 65-73 